\title{
Medical Image of the Week: Lymphangitic Carcinomatosis
}

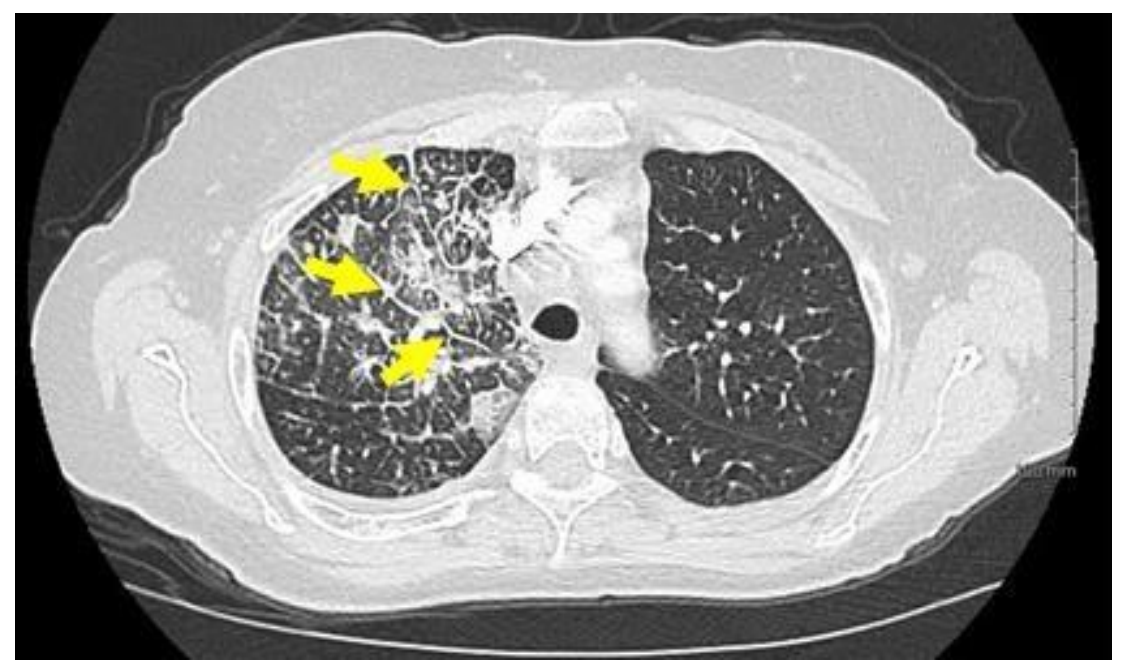

Figure 1. Mass like consolidation and interlobular septal thickening (arrows).

A 64-year-old woman, never-smoker, was evaluated for shortness of breath and left leg swelling. An abnormal initial chest X-Ray lead to computed tomography (CT) scan of the chest. She was also diagnosed with deep vein thrombosis (DVT) of her left leg.

CT of the chest with intravenous contrast showed a mass-like consolidation in the right upper lobe and thickening of the peripheral interlobular septa and of the bronchovascular bundles consistent with lymphangitic carcinomatosis (Figure 1). Endobronchial ultrasound (EBUS) guided transbronchial needle aspirations of the station $10 \mathrm{R}$ Lymph node were positive for adenocarcinoma of lung origin.

Lymphangitic carcinomatosis occurs when cancer cells spread along the pulmonary lymphatic system and result in thickening of the bronchovascular bundle, the interlobular septa, or both (1). Histopathologically, specimens show interlobular and subpleural interstitial desmoplastic thickening and obstruction of lymphatic vessels by tumor cells. It carries a poor prognosis.

Mohammad R. Dalabih, MBBS ${ }^{1}$ and Joshua Malo, MD²

${ }^{1}$ Pulmonary Consultants LLC, Tacoma, WA USA

${ }^{2}$ Division of Pulmonary, Allergy, Critical Care. And Sleep, University of Arizona College of Medicine, Tucson, AZ USA

\section{Reference}

1. Munk PL, Müller NL, Miller RR, Ostrow DN. Pulmonary lymphangitic carcinomatosis: CT and pathologic findings. Radiology. 1988 Mar;166(3):705-9. [CrossRef] [PubMed] 